\title{
Nanoscale Poroelasticity of the Tectorial Membrane Determines Hair Bundle Deflections
}

\author{
Jonathan B. Sellon, ${ }^{1, *}$ Mojtaba Azadi, ${ }^{2,3,4}$ Ramin Oftadeh, ${ }^{3,4}$ Hadi Tavakoli Nia, ${ }^{5}$ \\ Roozbeh Ghaffari, ${ }^{1}$ Alan J. Grodzinsky, ${ }^{3,4,6,7}$ and Dennis M. Freeman ${ }^{1,6}$ \\ ${ }^{1}$ Research Laboratory of Electronics, Massachusetts Institute of Technology, Cambridge, Massachusetts 02139, USA \\ ${ }^{2}$ School of Engineering, College of Science and Engineering, San Francisco State University, San Francisco, California 94132, USA \\ ${ }^{3}$ Center for Biomedical Engineering, Massachusetts Institute of Technology, Cambridge, Massachusetts 02139, USA \\ ${ }^{4}$ Department of Biological Engineering, Massachusetts Institute of Technology, Cambridge, Massachusetts 02139, USA \\ ${ }^{5}$ Massachusetts General Hospital, Harvard Medical School, Boston, Massachusetts 02114, USA \\ ${ }^{6}$ Department of Electrical Engineering and Computer Science, Massachusetts Institute of Technology, \\ Cambridge, Massachusetts 02139, USA \\ ${ }^{7}$ Department of Mechanical Engineering, Massachusetts Institute of Technology, Cambridge, Massachusetts 02139, USA
}

(Received 26 March 2018; revised manuscript received 14 October 2018; published 16 January 2019)

\begin{abstract}
Stereociliary imprints in the tectorial membrane (TM) have been taken as evidence that outer hair cells are sensitive to shearing displacements of the TM, which plays a key role in shaping cochlear sensitivity and frequency selectivity via resonance and traveling wave mechanisms. However, the TM is highly hydrated (97\% water by weight), suggesting that the TM may be flexible even at the level of single hair cells. Here we show that nanoscale oscillatory displacements of microscale spherical probes in contact with the TM are resisted by frequency-dependent forces that are in phase with TM displacement at low and high frequencies, but are in phase with TM velocity at transition frequencies. The phase lead can be as much as a quarter of a cycle, thereby contributing to frequency selectivity and stability of cochlear amplification.
\end{abstract}

DOI: 10.1103/PhysRevLett.122.028101

The tectorial membrane (TM) is widely believed to play a significant role in determining the remarkable frequency selectivity and sensitivity that are hallmarks of mammalian hearing by virtue of its resonance [1-5] and traveling wave properties [6-20]. In these conceptions of cochlear mechanics, cochlear hair bundles are envisioned as being either velocity or displacement sensitive depending on their anatomical position relative to the TM [4,21-23]. For instance, scanning electron microscopy studies have revealed imprints of the top row of outer hair cell $(\mathrm{OHC})$ cilia bundles on the undersurface of the TM [24-26]. As a result, the tips of $\mathrm{OHC}$ hair cell stereocilia are thought to be mechanically anchored and thus constrained to move with the TM [4,21-23,27-29].

Translation of mechanical pressure in the cochlea to hair bundle motion begins with displacement of the basilar membrane and lever motion of the TM. This lever motion then displaces the TM $\left(D_{\mathrm{TM}}\right)$ and generates a force on hair bundles $\left(F_{B}\right)$. The notion that $\mathrm{OHC}$ stereocilia and the TM are entrained to move in phase is predicated on the TM being a rigid body. However the TM is highly hydrated (97\% water by weight [30-34]), and the molecular

Published by the American Physical Society under the terms of the Creative Commons Attribution 4.0 International license. Further distribution of this work must maintain attribution to the author(s) and the published article's title, journal citation, and DOI. mechanism underlying the mechanical properties of the TM depends on both the elastic properties of the matrix and the viscous properties of the interstitial fluid [18,35]. Fluid flow through the nanoporous structure of the TM can directly affect the nanometer-scale motions of the hair bundles. Such fluid flow through the matrix and the separation of fluid flow and matrix movements can be directly measured at the nanoscale $[36,37]$. Thus, fluid flow through the nanoporous structure of the TM could directly affect the nanometer-scale motions of the hair bundles.

To investigate nanoscale interactions of the TM with hair bundles, we displace the TM dynamically at audio frequencies and measure the force applied to microscale spherical atomic force microscopy (AFM) probes [36-39] [Fig. 1(a)]. We observe elastic forces on the probe at both low and high frequencies and a surprising presence of viscous forces near transition frequencies. This hybrid velocity-displacement behavior (as opposed to displacement driven interaction) produces a frequency-dependent phase lead in the force a hair bundle would experience due to TM displacements (up to $\pi / 2 \mathrm{rad}$ ) that has not been previously described. Such a phase lead would tend to compensate for electrical, mechanical, and hydrodynamic phase delays that must be overcome for cochlear amplification.

To characterize TM mechanics on length scales relevant to the hair bundles, we used an atomic force cantilever attached to a spherical probe (diameter $1.5 \mu \mathrm{m}$ ) and engaged it on the undersurface of the TM. Quasistatic tip displacements into 
(a)

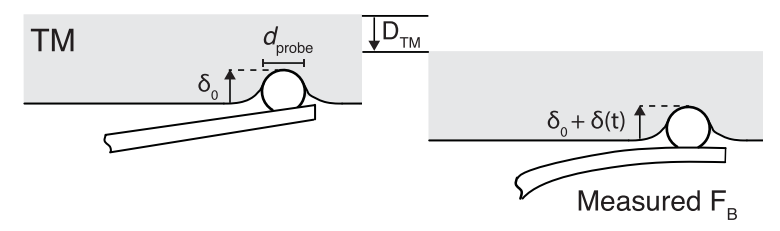

(c)

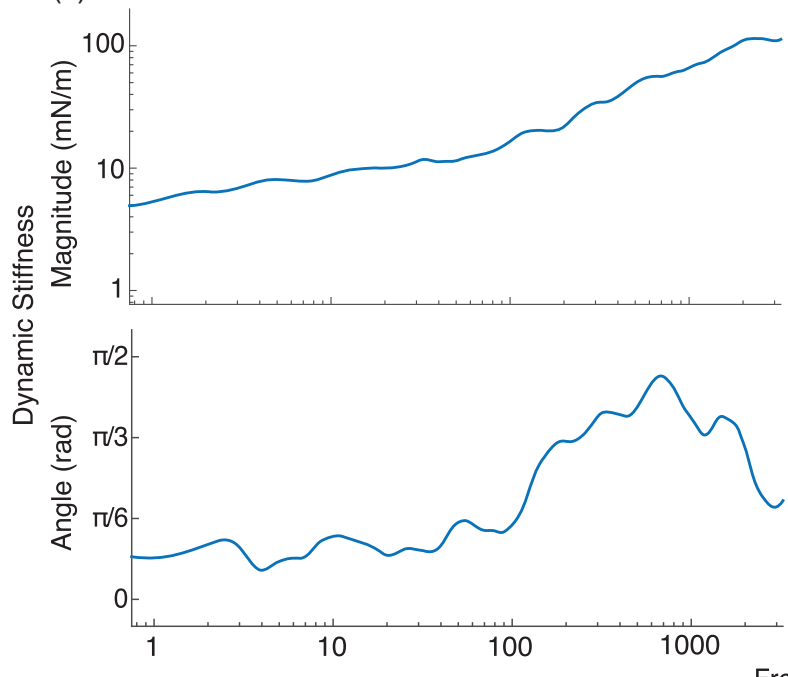

(b)

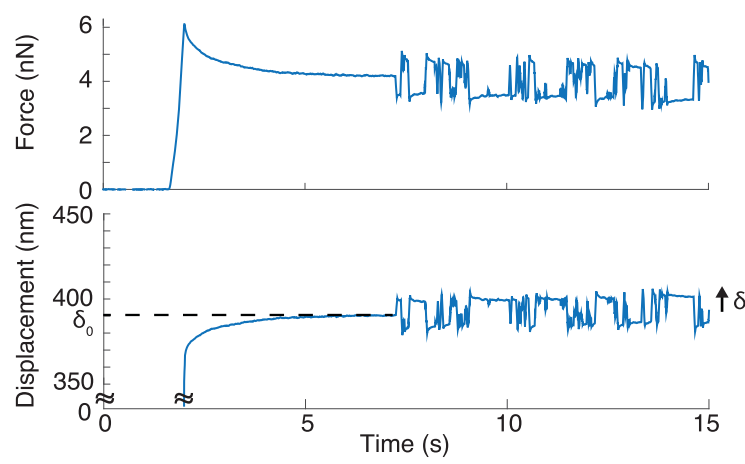

(d)

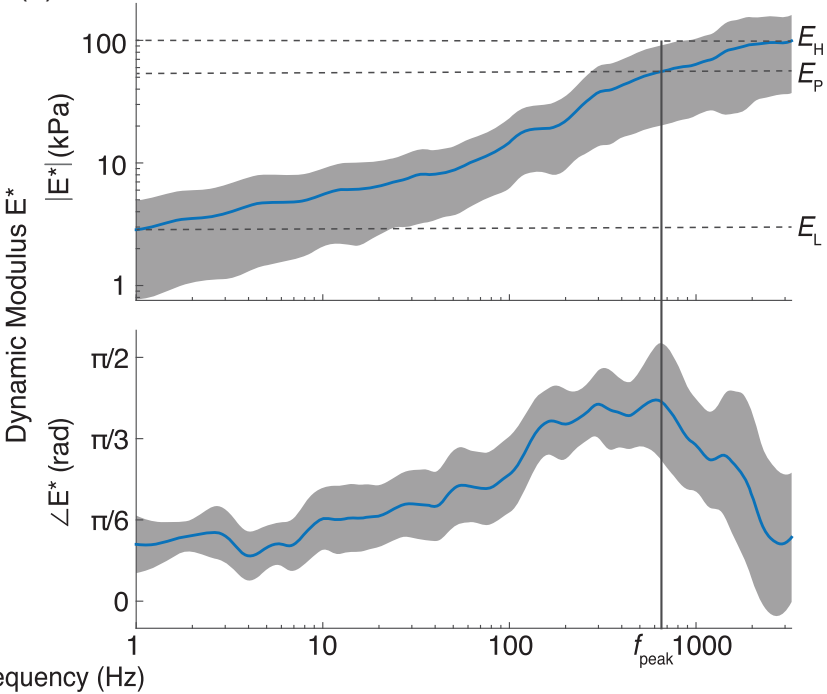

FIG. 1. Dynamic nanoindentation response of the TM. (a) Schematic depicting nanoscale dynamic indentations of the TM. To simulate TM-hair bundle interactions at length scales and frequencies relevant to hearing, we apply nanoscale displacements to the TM, $D_{\mathrm{TM}}$, at audio frequencies $(0.1-3 \mathrm{kHz})$ and measure the force $F_{B}$ applied to microscale probes of diameter $d_{\text {probe. }}$. The TM is initially indented by $\delta_{0}$ followed by stimulation at audio frequencies with amplitude $\delta$. (b) Example nanoscale indentation pattern for a typical TM sample. The deformation profile, which consists of an initial preindentation $\left(\delta_{0}=0.1-0.4 \mu \mathrm{m}\right)$, followed by wide bandwidth indentation with an amplitude $\delta \sim 15 \mathrm{~nm}$, was superimposed on the initial preindentation (bottom, see Supplemental Material [40]). The force on the probe due to this indentation pattern is then measured (top). (c) Magnitude (top) and phase (bottom) of the TM dynamic stiffness for one TM preparation and nanoindentation site with a indentation depth of $0.332 \mu \mathrm{m}$. (d) Magnitude $\left|E^{*}\right|$ (top) and phase $\angle E^{*}$ (bottom) of dynamic nanoindentation modulus. The mean (solid line) and SD (shaded area) are based on $n=9$ indentation sites on each of three TM preparations (blue curves). The modulus $E_{L}$ represents the low frequency elastic limit, $E_{P}$ represents the magnitude of the modulus at the frequency at which the phase peaks, $f_{\text {peak }}$, and $E_{H}$ represents the high frequency elastic limit. At low and high frequencies, the phase angle of the dynamic indentation response approaches zero, while near the transition frequency the phase angle approaches $\pi / 2$.

the TM applied at nanoscale depths $\left(\delta_{0}\right)$ (Fig. S1 [40]) yielded quasistatic stiffness estimates $(\sim 9 \mathrm{mN} / \mathrm{m}$, see Supplemental Material [40]) consistent with previous measurements [49]. These measurements were conducted for each indentation site prior to dynamic measurements.

To characterize the frequency-dependent mechanical properties of the TM, we next applied dynamic indentations at audio frequencies based on random binary sequences [Fig. 1(b); see Supplemental Material [40]] using the AFM probe. From the ratio of TM displacement to the measured force on the probe $\left(\left[F_{\text {osc }}(f) / \delta(f)\right]\right)$, we computed the dynamic stiffness of the TM for a wide range of stimulus frequencies (see Supplemental Material [40]). Figure 1(c) shows TM stiffness as a function of frequency for a typical TM sample.

To determine the dynamic material properties of the TM, we accounted for the size of the probe and indentation depth to measure the magnitude of the nanoscale dynamic complex indentation modulus,

$$
\left|E^{*}(f)\right|=\frac{F_{\text {osc }}(f)}{\delta(f)} \frac{1}{2\left(R \delta_{0}\right)^{1 / 2}},
$$

where $F_{\text {osc }}$ is the amplitude of the measured oscillatory force on the probe, $\delta(f)$ is the displacement applied to probe by the TM, $R$ is the probe radius, and $\delta_{0}$ is the 
indentation depth of the probe. Figure S2 [40] shows a single point measurement of $\left|E^{*}\right|$ of the TM. Figure 1(d) and Fig. S3 (top panels) [40] show $\left|E^{*}\right|$ modulus estimates across multiple TM preparations. At low frequencies, the nanoscale mechanical response approaches an asymptotic value corresponding to equilibrium elastic response, $E_{L}$ ( $2.85 \pm 2.08 \mathrm{kPa}$, mean $\pm \mathrm{SD}, n=9$ indentation regions). Compared to this low frequency nanoscale response, the quasistatic mechanical response [Fig. S1(b) [40] ] yielded a similar elastic modulus (4.92 $\pm 1.15 \mathrm{kPa}$, mean $\pm \mathrm{SD})$. For the dynamic response at high frequencies, the modulus approaches an asymptotic value, $E_{H}(96.1 \pm 61.1 \mathrm{kPa})$, significantly higher than $E_{L}$. These moduli match previous measurements of TM material properties $[6,9,12-14$, 17-19] (Tables S1 and S2 [40]).

In addition to $\left|E^{*}\right|$ magnitude, we also determined the phase $\angle E^{*}$ of the dynamic complex indentation modulus [Fig. 1(d) and Fig. S3, bottom panels [40] ], where 0 deg of phase accumulation would correspond to a purely stiff element and 90 deg would correspond to a purely viscous element. At both low and high frequencies, the phase $\angle E^{*}$ tends toward $\sim 20 \mathrm{deg}$. However, at transition frequencies, $\angle E^{*}$ increases to a peak of $\sim 74 \pm 21 \mathrm{deg}$ (mean $\pm \mathrm{SD}$ ), indicating a phase lead at those frequencies (where $f_{\text {peak }} \sim 620 \mathrm{~Hz}$ ). A transitional phase lead has not previously been reported to influence the motion of cochlear hair bundles, but has been reported to exist for poroelastic tissues [36-39].

Transitional phase leads are commonly observed for poroelastic materials because the energy dissipation in these materials is associated with viscous drag of fluid through the porous matrix. Relative motions between the fluid and matrix are greatest in the region near the contact between the TM tissue and probe tip. The thickness of the boundary layers in this region are frequency dependent and, as a result, there is a linkage between the size of the probe and the frequency dependence of the response. According to linear poroelasticity theory $[37,50]$, the peak frequency of the dynamic phase response at which $\angle E^{*}$ is a maximum is

$$
f_{\text {peak }} \propto \frac{k E}{d^{2}},
$$

where $E$ is the equilibrium modulus, $k$ is the hydraulic permeability, and $d=2 R \cos ^{-1}\left[\left(R-\delta_{0}\right) / R\right]$ is the characteristic length of that portion of the tissue that is in contact with the AFM tip. Thus, by probing the dependence of $f_{\text {peak }}$ on nanoindentation contact length $d$, we could determine whether poroelastic solid-fluid interactions dominate TM-hair bundle interactions as opposed to purely solid phase viscoelastic interactions, which would not scale with indentation contact length $d$. Furthermore, poroelastic theory [37] predicts that $f_{\text {peak }}$ is also inversely correlated with $\delta_{0}$, which we examine in Fig. S4 [40].
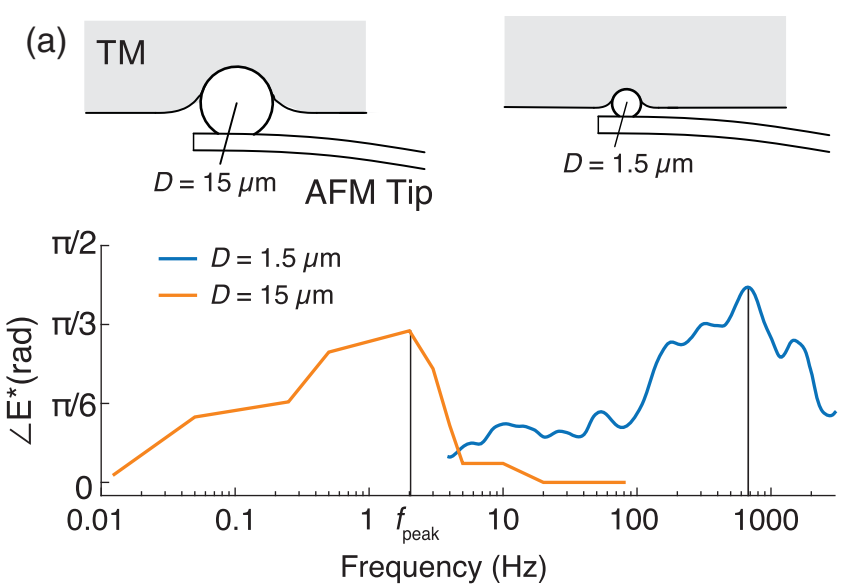

(b)

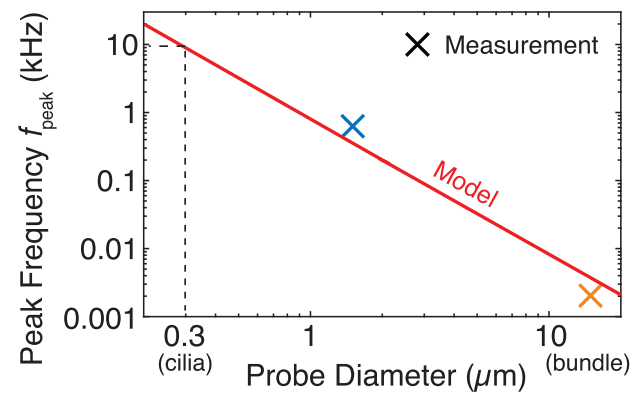

FIG. 2. Length-scale analysis of TM poroelasticity. (a) Poroelasticity theory predicts that $f_{\text {peak }} \propto\left(1 / d^{2}\right)$. Thus, increasing the contact area of the probe tip from a diameter of 1.5 to $15 \mu \mathrm{m}$ should decrease $f_{\text {peak }}$. Here, $f_{\text {peak }}$ was $\sim 620 \mathrm{~Hz}$ using a $\sim 1.5 \mu \mathrm{m}$ diameter probe tip for a typical apical region TM (average, $n=4$ indentations from preparation 2 in Fig. S2 [40]). In separate apical region preparations, a $15 \mu \mathrm{m}$ diameter probe tip resulted in an $f_{\text {peak }}$ of $\sim 2 \mathrm{~Hz}$ (average, $n=2$ indentation regions). Thus, reducing probe tip contact area increases $f_{\text {peak }}$ of the phase angle of the dynamic modulus. (b) $f_{\text {peak }}$ versus probe diameter for measurements in this study and that predicted for a single hair bundle cilium assuming a cilium diameter of $300 \mathrm{~nm}$ based on previous scanning electron microscopy imprints [24-26].

Previous TM shear impedance studies have also characterized TM anisotropy in the radial shear, longitudinal shear, and point indentations in the transverse directions over relevant frequency ranges [49,51]. These results show that TM radial shear impedance magnitude is greater by a factor of $\sim 2$ compared to the longitudinal shear and transverse impedances, with little to no apparent shift in the phase relation. These previous findings suggest that $\left|E^{*}\right|$ and $\angle E^{*}$ reported here are likely to be comparable (within a factor of 2) to those that would be driven by mechanical excitations in the radial direction.

To test the relationship between probe size and $f_{\text {peak }}$, we measured dynamic responses of the TM with probe tips of different sizes. For probes with $1.5 \mu \mathrm{m}$ diameter, $f_{\text {peak }}$ is $\sim 620 \mathrm{~Hz}$. For probes with $15 \mu \mathrm{m}$ diameter, $f_{\text {peak }}$ is $\sim 2 \mathrm{~Hz}$ (Fig. 2). $f_{\text {peak }} \propto\left(1 / d^{2}\right)$, which would suggest that a probe with diameter on the order of a cilium (diameter of 
$300 \mathrm{~nm}$ [24-26]) would yield an $f_{\text {peak }}$ in the auditory range. Furthermore, for the lowest frequency measurement with the $15 \mu \mathrm{m}$ probe $(0.01 \mathrm{~Hz}), f_{\text {peak }}$ approaches 0 deg versus the near 20 deg lead observed for the lowest frequency measurement with the $1.5 \mu \mathrm{m}$ probe $(1 \mathrm{~Hz})$. Decreased indentation depth also shifts $f_{\text {peak }}$ to higher frequencies and thus the low frequency asymptote can also approach $0 \mathrm{deg}$ for the $1.5 \mu \mathrm{m}$ probe (Fig. S4 [40]). The off-transition frequency lead has previously been attributed to tissue viscoelasticity [38], whereas the phase lead at the transition frequency has been attributed to tissue poroelasticity [37].

Using a previously developed fibril reinforced poroelastic finite element model [37] (see Supplemental Material [40]), we can determine the hydraulic permeability $k$ of the TM. Based on the relationship in Eq. (2) and best fits to the poroelastic model (to $E_{L}$ and $f_{\text {peak }}$ ), apical regions of the TM have $k \sim 7.44 \pm 3.88 \times 10^{-14} \mathrm{~m}^{4} / \mathrm{N}$ s. This result shows that the TM is approximately an order of magnitude more permeable to fluid flow than other poroelastic tissues such as cartilage [37]. Furthermore, the high hydraulic permeability of the TM is consistent with the highly hydrated state of the TM, which is $97 \%$ water by weight [30-34], as well as with previous measurements of permeability for synthetic hydrogels (Table S3 [40]).

Based on the position of $\mathrm{OHC}$ stereocilia relative to the overlying TM, deflection of $\mathrm{OHC}$ bundles have previously been thought to be proportional to TM displacement. Thus, $\mathrm{OHCs}$ were classified as displacement sensors to relative motions of the reticular lamina and TM [22]. This view assumes that $\mathrm{OHC}$ cilia are directly embedded in a rigid TM and motions of cilia would follow exactly with those of the TM. Here we see that the TM is not a rigid body, but rather a poroelastic gel whereby displacements of the TM are resisted by displacements of hair bundles. This resistance causes a local deformation of the TM such that hair bundles do not move in proportion to the gross motions of the TM. Rather, the resistance from hair bundles generates local deformation of TM [Fig. 3(a)]. At both low and high frequencies, the TM exerts near-elastic forces on the OHC stereocilia. In contrast, at transition frequencies, $\mathrm{OHC}$ stereocilia are entrained by fluid-solid viscous dissipation forces associated with the velocity of fluid through the TM's porous structure [Fig. 3(b); Video S1 [40] ], causing the torque on the bundle $\left(\tau_{B}\right)$ to be out of phase with bundle displacement $\left(x_{B}\right)$ [Fig. 3(c)]. Thus, the poroelastic properties of the TM give rise to a frequency range over which OHCs respond to fluid velocity rather than displacement sensors in response to sound.

Cochlear amplification is thought to rely on a positive feedback loop in the cochlea that amplifies the basilar membrane traveling wave. To achieve high sensitivity and sharp frequency selectivity, cochlear models assume that force generation by the OHCs must be in synchrony with the basilar membrane wave over a narrow frequency range. In order to maintain this time synchrony, the cochlea must (a)

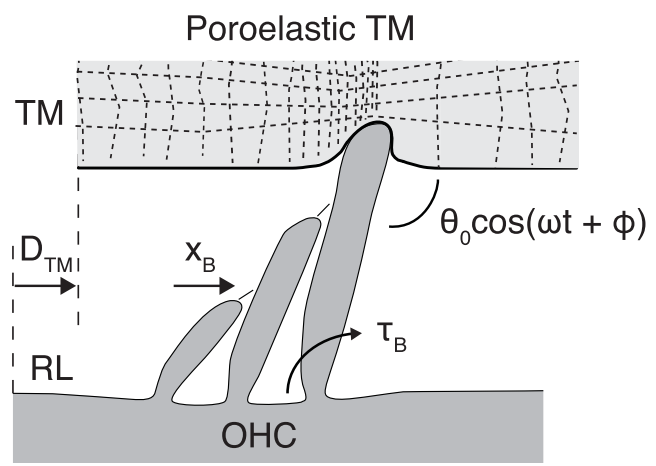

(b)

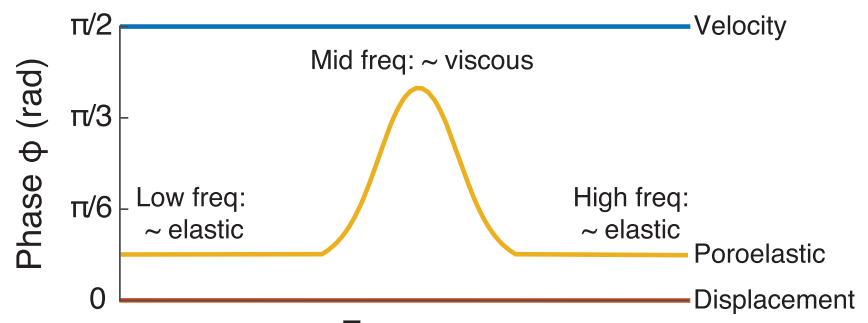

(c)

Frequency

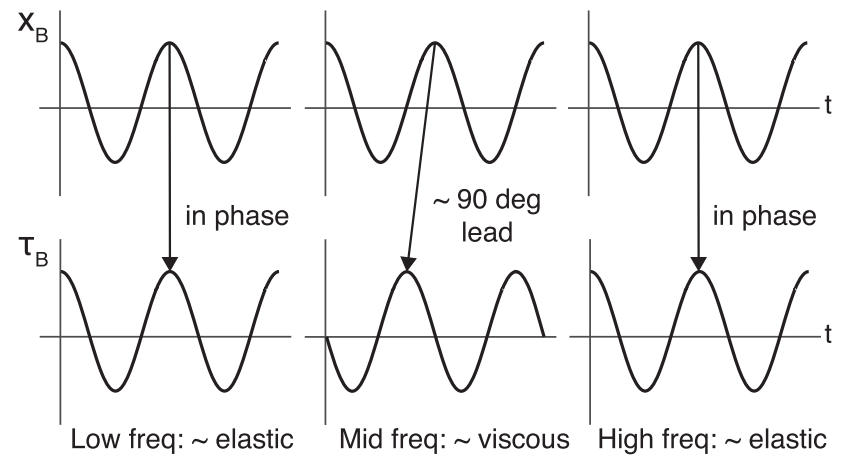

FIG. 3. Schematic drawing of OHC stereocilia and TM interactions. (a) Deflection of the TM $\left[D_{\mathrm{TM}}=D_{0} * \cos (\omega t)\right]$ induces displacements of hair bundles $\left(x_{B}\right)$ and torque on the bundle $\left(\tau_{B}\right)$ by applying a force with an angle $\theta$ with respect to the reticular lamina (RL). Given that the TM is porous, solid-fluid interactions of the TM's matrix cause the TM to be compressed in resistance to force applied by the cilia bundle and stretched on the side opposite to the stimulation. These TM-hair bundle interactions at low, middle, and high frequencies are animated in Video S1 [40]. (b) Transfer function characteristics for freestanding hair bundles experiencing purely viscous forces (blue curve), elastic forces (red curve), and poroelastic forces based on results presented in this Letter (yellow curve). Phase differences between the displacement of the TM and the force applied to the hair bundle $\left[\phi\right.$, where $\left.\theta=\theta_{0} * \cos (\omega * t+\phi)\right]$ as a function of frequency suggest that $\mathrm{OHC}$ bundles tend toward displacement sensing behavior at low and high frequencies, but velocity sensing behavior at middle frequencies. (c) Depiction of phase relationships between $x_{B}$ and $\tau_{B}$ at low frequencies, transition frequencies, and high frequencies.

overcome many sources of phase lag (e.g., fluid inertia in the subtectorial space, the OHC membrane time constant, and ciliary dynamics) [1-5]. Our results show that TM 
poroelasticity could introduce significant phase lead in the force that a bundle experiences at transition frequencies versus very low or high stimulation frequencies. This phase lead may be one of many factors needed to drive positive feedback in cochlear models. Furthermore, this phase lead is dependent only on the relation between the TM and the $\mathrm{OHC}$ bundle, regardless of the mechanisms that drive TM motion. Thus, TM poroelastic properties may be required to overcome sources of phase lag in the cochlea and to achieve high sensitivity in mammalian hearing.

Recent in vivo work highlights the complex motions of the TM, basilar membrane, and reticular lamina that result in sharp auditory tuning [16,52-54]. In addition to microscale motions of these structures, we observe that the nanoscale interactions of the TM and OHC stereocilia may also contribute to sharp frequency tuning. Our results show that TM poroelasticity generates $\mathrm{OHC}$ stereociliary deformations with phase accumulation over a narrow frequency range [Figs. 1(c) and 1(d)] and in the auditory range for cilia-sized point deflections [Fig. 2(b)]. Furthermore, we see two orders of magnitude difference in $f_{\text {peak }}$ between measurements with the 1.5 and $15 \mu \mathrm{m}$ probes [Fig. 2(a)], and our model predicts that the characteristic frequency of this transitional phase lead would be in the auditory range for a single cilia [Fig. 2(b)]. In vivo, the exact characteristic frequency of this transitional phase lead would arise from multiple factors, including the depth of the embedded cilia in the TM, the amount of fluid coupling between individual cilia in a hair bundle, and the poroelastic material properties of the TM at the attachment point. Given that the predicted transition frequency for cilia-sized indentations is in the auditory range, sharpness of cochlear tuning may thus be driven, in part, by the poroelastic interactions of the TM and $\mathrm{OHC}$ stereocilia.

This work was supported by National Institutes of Health Grant No. R01-DC000238 (J. B. S., R. G., and D. M. F.) and No. F32-CA216944-01 (H. T. N.), National Science Foundation Grant No. CMMI-1536233 (M. A., R. O., H. T. N., and A. J. G.), and National Science Foundation Graduate Research Fellowship under Grant No. 1122374 (J. B. S.).

*sellon@mit.edu

[1] J. B. Allen, J. Acoust. Soc. Am. 68, 1660 (1980).

[2] F. Mammano and R. Nobili, J. Acoust. Soc. Am. 93, 3320 (1993).

[3] J. J. Zwislocki, J. Acoust. Soc. Am. 67, 1679 (1980).

[4] S. T. Neely and D. O. Kim, Hear. Res. 9, 123 (1983).

[5] S. T. Neely and D. O. Kim, J. Acoust. Soc. Am. 79, 1472 (1986).

[6] R. Ghaffari, A. J. Aranyosi, and D. M. Freeman, Proc. Natl. Acad. Sci. U.S.A. 104, 16510 (2007).

[7] R. Gueta, J. Levitt, A. Xia, O. Katz, J. S. Oghalai, and I. Rousso, Biophys. J. 100, 2530 (2011).
[8] R. Gueta, E. Tal, Y. Silberberg, and I. Rousso, J. Struct. Biol. 159, 103 (2007).

[9] R. Gueta, D. Barlam, R. Z. Shneck, and I. Rousso, Proc. Natl. Acad. Sci. U.S.A. 103, 14790 (2006).

[10] N. Gavara and R. S. Chadwick, PLoS One 4, e4877 (2009).

[11] C. P. Richter, G. Emadi, G. Getnick, A. Quesnel, and P. Dallos, Biophys. J. 93, 2265 (2007).

[12] N. Gavara and R. S. Chadwick, Nat. Methods 7, 650 (2010).

[13] B. Shoelson, E. K. Dimitriadis, H. Cai, B. Kachar, and R. S. Chadwick, Biophys. J. 87, 2768 (2004).

[14] R. Ghaffari, A. J. Aranyosi, G. P. Richardson, and D. M. Freeman, Nat. Commun. 1, 96 (2010).

[15] J. Meaud and K. Grosh, J. Acoust. Soc. Am. 127, 1411 (2010).

[16] H. Y. Lee, P. D. Raphael, J. Park, A. K. Ellerbee, B. E. Applegate, and J. S. Oghalai, Proc. Natl. Acad. Sci. U.S.A. 112, 3128 (2015).

[17] G. P. Jones, V. A. Lukashkina, I. J. Russell, S. J. Elliott, and A. N. Lukashkin, Biophys. J. 104, 1357 (2013).

[18] J. B. Sellon, R. Ghaffari, S. Farrahi, G. P. Richardson, and D. M. Freeman, Biophys. J. 106, 1406 (2014).

[19] J. B. Sellon, S. Farrahi, R. Ghaffari, and D. M. Freeman, Proc. Natl. Acad. Sci. U.S.A. 112, 12968 (2015).

[20] J. B. Sellon, R. Ghaffari, and D. M. Freeman, Biophys. J. 112, 1059 (2017).

[21] H. Davis, Ann. Otol., Rhinol., Laryngol. 67, 789 (1958).

[22] P. Dallos, M. C. Billone, J. D. Durrant, C. Wang, and S. Raynor, Science 177, 356 (1972).

[23] M. Billone and S. Raynor, J. Acoust. Soc. Am. 54, 1143 (1973).

[24] D. Lim, Archives of Otolaryngology 96, 199 (1972).

[25] M. Lenoir, J.-L. Puel,and R. Pujol, Anatomy and Embryology 175, 477 (1987).

[26] L. R. Andrade, F. T. Salles, M. Grati, U. Manor, and B. Kachar, J. Struct. Biol. 194, 139 (2016).

[27] J. H. Nam, Hear. Res. 360, 31 (2018).

[28] J. Holt, J. T. Xue, A. Brichta, and J. Goldberg, J. Neurophysiol. 95, 428 (2006).

[29] I. S. Curthoys, V. Vulovic, A. M. Burgess, L. Sokolic, and S. C. Goonetilleke, Hear. Res. 331, 131 (2016).

[30] G. P. Richardson, I. J. Russell, V. C. Duance, and A. J. Bailey, Hear. Res. 25, 45 (1987).

[31] I. Thalmann, G. Thallinger, T. H. Comegys, E. C. Crouch, N. Barrett, and R. Thalmann, Laryngoscope 97, 357 (1987).

[32] I. Thalmann, Connect. Tissue Res. 29, 191 (1993).

[33] H. Suzuki, Y. C. Lee, M. Tachibana, K. Hozawa, H. Wataya, and T. Takasaka, Hear. Res. 60, 45 (1992).

[34] R. Killick, P. K. Legan, C. Malenczak, and G. P. Richardson, J. Cell Biol. 129, 535 (1995).

[35] K. Masaki, T. F. Weiss, and D. M. Freeman, Biophys. J. 91, 2356 (2006).

[36] L. Han, E. H. Frank, J. J. Greene, H. Lee, H. K. Hung, A. J. Grodzinsky, and C. Ortiz, Biophys. J. 100, 1846 (2011).

[37] H. T. Nia, L. Han, Y. Li, C. Ortiz, and A. Grodzinsky, Biophys. J. 101, 2304 (2011).

[38] B. K. Connizzo and A. J. Grodzinsky, J. Biomech. 54, 11 (2017).

[39] M. Azadi, H. T. Nia, S. J. Gauci, C. Ortiz, A. J. Fosang, and A. J. Grodzinsky, J. Biomech. 49, 1634 (2016). 
[40] See Supplemental Material at http://link.aps.org/ supplemental/10.1103/PhysRevLett.122.028101 for detailed methods, four supplemental figures, three tables, and an animated model, which includes Refs. [41-48].

[41] D. Shah, D. M. Freeman, and T. F. Weiss, Hear. Res. 87, 187 (1995).

[42] H. T. Nia, L. Han, I. S. Bozchalooi, P. Roughley, K. YoucefToumi, A. J. Grodzinsky, and C. Ortiz, ACS Nano 9, 2614 (2015).

[43] R. E. Mahaffy, S. Park, E. Gerde, J. Käs, and C. K. Shih, Biophys. J. 86, 1777 (2004).

[44] I. S. Bozchalooi, K. Youcef-Toumi, D. J. Burns, and G. E. Fantner, Rev. Sci. Instrum. 82, 113712 (2011).

[45] L. Ljung, System Identification (John Wiley \& Sons, Inc., New Jersey, 1998).

[46] M. L. Oyen, Int. Mater. Rev. 59, 44 (2014).
[47] D. G. Strange and M. L. Oyen, J. Mech. Behav. Biomed. Mater. 11, 16 (2012).

[48] H. T. Nia, I. S. Bozchalooi, Y. Li, L. Han, H. Hung, E. Frank, K. Youcef-Toumi, C. Ortiz, and A. Grodzinsky, Biophys. J. 104, 1529 (2013).

[49] C. C. Abnet and D. M. Freeman, Hear. Res. 144, 29 (2000).

[50] A. J. Grodzinsky, Fields, Forces and Flows in Biological Systems (Garland Science, New York, 2011).

[51] J. W. Gu, W. Hemmert, D. M. Freeman, and A. J. Aranyosi, Biophys. J. 95, 2529 (2008).

[52] J. B. Dewey, A. Xia, U. Müller, I. A. Belyantseva, B. E. Applegate, and J. S. Oghalai, Cell Rep. 23, 2915 (2018).

[53] W. He, D. Kemp, and T. Ren, eLife 7, e37625 (2018).

[54] H. Y. Lee, P. D. Raphael, A. Xia, J. Kim, N. Grillet, B. E. Applegate, A. K. E. Bowden, and J. S. Oghalai, J. Neurosci. 36, 8160 (2016). 